\title{
GESTIÓN Y COMPETITIVIDAD EN LA ERA DE LA GLOBALIZACIÓN ${ }^{1}$
}

Francisca Bouby Tolentino*

E-mail:f_bouby@yahoo.com

\section{RESUMEN}

El objetivo de este trabajo es realizar un análisis de lo que significa hacer gestión en la nueva era del cambiante sistema capitalista, caracterizado por la plena vigencia de la economía de mercado y la globalización acelerada.

Hacer gestión en un país en desarrollo es muy diferente que hacer gestión en un país desarrollado. La globalización de los sistemas productivos permite clasificar a los diferentes países del mundo según el peso relativo de sus respectivas fuerzas de trabajo, dependiendo, fundamentalmente de la competitividad a nivel mundial que estas fuerzan tienen.

Sobre esta base se estima que en los países desarrollados que integran el Primer Mundo, la mayoría de la fuerza laboral tiene el nivel de competitividad adecuado con los estándares internacionales. De otro lado, en los países en desarrollo de América Latina, Asia Oriental y el antiguo mundo soviético, que conforman el Tercer Mundo, no obstante tener ciertas fuerzas laborales competitivas, no podrán absorber con facilidad al grueso de la fuerza laboral que provienen de sus sectores económicos rurales e informales. Esta posición se refuerza en el hecho de que los niveles de competitividad internacional se elevan continuamente de acuerdo con los avances de la tecnología.

Palabras clave: Gestión, competitividad, globalización, fuerzas productivas.

\section{ABSTRACT}

The objective of this work is to carry out an analysis to manage the new era of the changing capitalist system, characterized by the full validity of the market economy and the quick globalization.

To manage a country in development is different from managing a developed country. The globalization of the productive systems, allows the classification of the different countries in the world according to the relative weight of its respective work forces, depending, fundamentally on the world level competitiveness that these forces have.

On this base it is considered that in the developing countries that integrate the First World, most of the labor force has the appropriate competitiveness level with the international standards. On the other hand, the Latin American countries in development as well as the Oriental Asia and the old Soviet world that conform the Third World, will not be able to absorb most of the labor force that come from the rural and informal economic sector. This position is reinforced in the fact that the levels of international competitiveness rise continually according to the advance of the technology.

Keywords: Administration, competitiveness, globalization, productive forces.

* Doctora en Economía. Magíster en Economía con mención en Política Económica. Economista. Profesora Principal e Investigadora de la Facultad de Ciencias Administrativas (UNMSM). 


\section{INTRODUCCIÓN}

La realidad de los países donde el desarrollo de la ciencia y tecnología ha quedado postergado indica que éstos no se encuentran en condiciones de acceder a estos avances cuyo cambio es vertiginoso; por lo tanto, sus niveles de competitividad guardan un rezago en relación con los estándares internacionales.

Los países no industrializados o no competitivos de África y el mundo árabe, que conforman el Cuarto Mundo ${ }^{2}$, sufren una situación todavía peor: están totalmente excluidos de la industrialización y la carencia de una revolución agraria conduce a un sistema de mayor marginación social, donde los pobres y las masas campesinas llevan la peor parte.

La persistencia de querer hacer funcionar al mundo como un gran mercado bajo la utopía liberal viene produciendo resultados nefastos frente a los cuales surgen grandes responsabilidades de parte de los intelectuales, cuyas críticas deben orientarse sistemáticamente tanto al discurso superficial del carácter ineludible de la globalización, así como al discurso actual de que la expansión del mercado conduce al progreso social y a la democracia, y que dificultades como la pobreza, el desempleo y la marginación social, son meramente transitorias.

En los últimos años ha surgido en la mesa de debate nuevas posibilidades históricas de desarrollo económico, donde los conceptos de coordinación y aprendizaje ocupan un lugar central en el estudio de la reinserción internacional, como respuesta al surgimiento de un nuevo paradigma tecnológico y a profundo cambio estructural y espacial en la economía global, que determinaron el agotamiento de las viejas formas de integración internacional. La reinserción internacional de las economías en desarrollo puede ser el eje en torno al cual se despliegan oportunidades de desarrollo nacional.

$Y$ es que el conjunto de cambios que trae la globalización, provocados por el régimen de acumulación, se expresan a su vez en el modo de operar de los Estados y de las unidades privadas de la sociedad mundial. Actualmente el Estado nacional no coincide con la extensión de la sociedad ni con la economía nacional, por lo que la globalización designa los cambios que se van suscitando en la sociedad ${ }^{3}$.
La regionalización surge como respuesta a las nuevas condiciones de acumulación que enfrentan los Estados, que se ven en la necesidad de cambiar sus políticas económicas, a pesar de no existir compatibilidad entre las políticas macroeconómicas de los integrantes de los bloques creados. De otro lado la globalización financiera ha hecho extremadamente sensibles a los mercados emergentes, por lo que un adecuado manejo de la política cambiaria es una línea de defensa frente a ataques especulativos que amenazan permanentemente con la modificación del régimen cambiario.

Sin embargo, según el modelo de Programación Financiera desarrollado por el Fondo Monetario Internacional (FMI), dada la existencia de una tendencia a un exceso en el gasto público de parte de los países en desarrollo y a la carencia de independencia de los Banco Centrales, hay una presión permanente sobre las reservas internacionales, precipitándose las crisis de balanza de pagos.

Una de las críticas fuertes al modelo neoliberal es que teóricamente no aborda el problema del desempleo, pues parte del supuesto de pleno empleo de los factores productivos. Tampoco contempla el problema del financiamiento de las Micro, Pequeñas y Medianas empresas (MIPYMES), siendo que éstas justamente son las mayores generadoras del empleo en los países en desarrollo. Finalmente, surge un serio cuestionamiento a los Bancos Centrales de los países en desarrollo por su falta de independencia en el manejo de la política monetaria.

El conjunto de estos factores abordados en este trabajo son los que condicionan el desarrollo de las fuerzas productivas en los países en desarrollo, y con ello limitan los niveles de competitividad y la capacidad de hacer gestión de empresas en países en desarrollo como los latinoamericanos.

\section{GLOBALIZACIÓN DE LOS SISTEMAS PRODUC- TIVOS}

Las economías latinoamericanas vienen sufriendo profundos cambios desde la década de los ochenta, debido a la aplicación de programas de ajuste estructural, y también como parte de la acción de dos tendencias que dominan el escenario internacional, como son la globalización y la regionalización. 
Entendemos por globalización al actual proceso de crisis, reestructuración y cambio mundial que ha venido manifestándose desde la década de los setenta, y que abarca a todas las economías a nivel mundial. Dado que el avance de la globalización implica la integración de actividades económicas dispersas geográficamente a nivel internacional, cabe precisar que su evolución se ha dado esencialmente a través de la internacionalización del capital lo cual, a su vez, ha generado la conformación de grandes regiones económicas dentro de la economía mundial. La complementariedad de los mercados nacionales da sentido a la reformulación de la competitividad de las macroregiones; esto es, da cuerpo a la tendencia hacia la regionalización presente en la economía global ${ }^{4}$.

El surgimiento de bloques económicos tales como el Tratado de Libre Comercio de América del Norte (TLCAN), el Mercado Común Centroamericano (MCCA), el Mercado Común del Sur (MERCOSUR), Comunidad Andina de Naciones (CAN), etc., dan cuenta de un proceso de alta competencia.

Asimismo, la integración de los países de la región latinoamericana al mercado internacional conlleva a modificar sus políticas estratégicas de comercio e inversión; de igual manera muchas de las instituciones que estaban operando bajo el modelo de crecimiento anterior, el de Industrialización por Sustitución de Importaciones (ISI), tienen que cambiar al nuevo modelo de Industrialización Orientada a las Exportaciones (IOE).

El proceso de globalización genera muchas interpretaciones, varias de ellas contradictorias. Así, de un lado, se postula que si se logran reproducir a nivel global las condiciones de aplicación de las políticas económicas instrumentadas en los países desarrollados considerados exitosos, es posible restablecer la economía de pleno empleo a nivel global, lo cual implica la aceptación del ejercicio de una política estatal general. Sin embargo, dado que no existe un Estado global con esta facultad, la economía no se constituye a escala mundial. De otro lado, el hecho de que el Estado de bienestar sólo se logra restaurar en algunos países a nivel global, hace que el sistema capitalista se desarrolle teniendo como base mecanismos de reproducción de una polarización y exclusión cada vez mayores, cuyo análisis trasciende el campo económico a lo social, cultural y político ${ }^{5}$.
En consecuencia, la inserción del territorio en segmentos específicos de la cadena de valor no es tan sencilla; se considera necesario generar condiciones competitivas para el sector productivo de las economías, a partir de los cuales se generan oportunidades y retos sistémicos de políticas, que van mucho más allá de las políticas a nivel macro, micro, meso y metaeconómicas ${ }^{6}$.

De allí que sea necesario que las economías consideren la generación de una batería de instrumentos y políticas para mejorar los niveles de competitividad y de inserción territorial al proceso de globalización. Es decir, incorporando aspectos de territorialidad, cadenas de valor y competitividad sistémica, en concordancia con un concepto aún más complejo como es el de la competitividad global ${ }^{7}$.

Desde los setenta y principalmente desde los ochenta, las empresas transnacionales (ET) han venido transfiriendo una parte creciente de segmentos de sus cadenas mercantiles globales (global commodity chains) ${ }^{8}$. Los cambios en la estructura productiva global se fueron dando en respuesta a la creciente flexibilización de la demanda, inicialmente en sectores industriales de punta como automotriz, electrónica y confección. En un contexto de apertura en los mercados de capitales y de bienes y servicios tanto a nivel de países en desarrollo como desarrollados, acompañados de mejoras en el transporte, y la revolución informática y de los medios de comunicación electrónicos, las ET transfieren territorialmente segmentos de la cadena de valor agregado de sus actividades ${ }^{9}$. En América Latina las ET exportaban sus productos a la región o las producían en el continente hasta antes de los ochenta; a partir de esta década, dichas empresas fueron integrando segmentos de la cadena de valor como parte de la estrategia y red global de productos finales.

El proceso de transferencia de cadenas de valor en diversos territorios, propiciado por la globalización, ha generado el proceso de subcontratación (outsourcing) a nivel global, inicialmente en la esfera productiva, luego en la de distribución y finalmente en la esfera de comercialización. Este proceso ha permitido que algunos territorios se especialicen en procesos de segmentos específicos de la cadena de valor, tales como materia prima, ensamble y sub ensamble, producción de partes y componentes, 
investigación y desarrollo, mercadeo, servicios post venta, etc. Dichos procesos requieren del desarrollo del comercio internacional con el objeto de integrar los segmentos a un producto o servicio final. A partir de los noventa surge el concepto de grandes clientes ${ }^{10}$, referido a la exigencia de que los subcontratistas se hagan directamente responsables -en forma integral- de los costos, inventarios, financiamiento, calidad, cantidad, insumos y del propio proceso, de transformación, así como del empaque y envío al consumidor final. Estos procesos, conocidos como "paquete completo" permitirían un mayor grado de integración y de apropiación del valor agregado por parte de los respectivos territorios, generando a su vez nuevos retos tanto a los sub contratistas como a los territorios donde se realizan estos procesos.

Otro aspecto relevante es el de la eficiencia colectiva $^{11}$ para los territorios y su papel para comprender el comportamiento positivo de los agrupamientos o clusters. La eficiencia colectiva y la conformación de redes interempresa en territorios específicos son justamente la base de su competitividad global. Otro concepto que se suma al análisis es el tipo de gobernabilidad de las cadenas (chain governance) que determinan la integración al mercado mundial. Así, cuanto mayor la verticalidad y el control de un reducido grupo de clientes y/o compradores, menor será el potencial de escalamiento local y de difusión y aprendizaje. De acuerdo con Ernst,

“... el escalamiento industrial necesita de una fuerte base de conocimiento... sustanciales inversiones en activos de largo plazo, tales como habilidades y capacidades de innovación e investigación especializadas...en países donde la estructura industrial doméstica ofrezca incentivos limitados a las empresas para invertir en estos activos de largo plazo, el potencial de escalamiento continuará limitado"12.

Por el contrario, cuanto mayor sea el número de clientes y menor la dependencia de estándares impuestos por parte de las empresas líderes, mayores serán las opciones de integración, coordinación, cooperación, difusión, aprendizaje y escalamiento local e interempresarial; es decir, de conformación de un tejido de redes interempresa territorial.

De lo anterior se deduce que el debate sobre competitividad y la generación de condicio- nes competitivas para el sector productivo implican políticas territoriales de capacitación; difusión de tecnología; de generación de capacidades; sistemas de incentivos en general; de fomento a la micro, pequeña y mediana empresa; investigación y desarrollo; del marco regulatorio y de competencia; incorporación de empresas proveedoras y la relación público-privada, entre $\operatorname{otros}^{13}$. Al respecto, Rodrick diferencia la relevancia de la calidad de las instituciones de la importancia del contexto institucional, incluso por encima de las políticas comerciales ${ }^{14}$.

En consecuencia, para comprender en la actualidad el cabal significado de la competitividad se deben examinar sus dimensiones sistémica, territorial y de segmentos de cadenas de valor, lo cual llevará a entender a nivel micro, meso, macro y meta económico la racionalidad de los encadenamientos mercantiles globales y la creciente flexibilización productiva, las redes intra e interempresa, los requerimientos de competitividad de los segmentos de la cadena global, y la calidad de las instituciones desde los respectivos territorios y su población ${ }^{15}$. Desde esta perspectiva es importante considerar en el análisis del proceso de globalización, por encima de la especialización en la manufactura y ensamble de los mismos, el desarrollo de capacidades de tecnología y de diseño de productos en territorios, potenciando sus opciones de escalamiento e integración a las cadenas globales competitivas. Entendemos como proceso de escalamiento a la integración y apropiación a superiores segmentos de la cadena y, por lo tanto, de mayor valor agregado, el mismo que puede darse a nivel de productos, procesos intra o intercadena ${ }^{16}$.

De allí que los efectos del proceso de globalización en términos socioeconómicos y territoriales, pueden generar los denominados procesos de endogeneidad territorial ${ }^{17} \mathrm{o}$ de polarización, lo que depende de las condiciones financieras estables que brinden autonomía a la gestión macroeconómica de las economías nacionales; sin embargo, la apertura creciente de las economías en desarrollo restringe dicha autonomía para aplicar medidas de políticas económicas nacionales apropiadas, dado que los movimientos de capital que han acompañado a la globalización financiera se han asociado con una mayor inestabilidad, tal como se analiza en el acápite siguiente. 


\section{GLOBALIZACIÓN FINANCIERA}

Los movimientos de capital que vienen acompañando a la globalización financiera se asocian con una mayor inestabilidad en las economías en desarrollo, y se ha aceptado que una mayor apertura financiera puede restringir la autonomía de la gestión macroeconómica.

La mayor movilidad financiera se constata con el aumento desbordante del mercado de divisas, como consecuencia de una mayor integración de los mercados financieros, especialmente de los países desarrollados. El incremento en la movilidad del capital ha traído como resultado un proceso de inversión más dinámico a nivel global. Sin embargo, dicha movilidad ha resultado también en una mayor inestabilidad, dado lo especulativo de la inversión de cartera que es capaz de desatar una crisis en cualquier país que no observe la disciplina financiera que impone la globalización, tal como aconteció a finales de 1994 en México ${ }^{18}$.

Los efectos de la inestabilidad resultante sobre los países en desarrollo se dejaron sentir por diversas vías. Buena parte de sus exportaciones e importaciones, así como de sus obligaciones del servicio de la deuda, se denominan en las principales divisas. Por causa de los desequilibrios entre las monedas en que vienen expresadas las salidas y entradas, la posición de las balanzas de pagos de los países en desarrollo suele ser sensible a los movimientos relativos de las diferentes monedas y a las fluctuaciones de los tipos de interés más importantes. Asimismo, el incremento de las obligaciones del servicio de la deuda como consecuencia de los tipos de interés más altos, coincide muchas veces con menores posibilidades de exportación, muy relacionadas también con un dinero más caro.

En consecuencia, la liberalización financiera global habría resultado exitosa para las economías desarrolladas, principalmente Estados Unidos pero, en cambio, ha aumentado la vulnerabilidad de las economías en desarrollo, principalmente de las denominadas "emergentes" lo cual demuestra la incapacidad del Fondo Monetario Internacional (FMI) para frenar la crisis y evitar sus efectos en cadena ${ }^{19}$.

\section{EL FINANCIAMIENTO EN LA ECONOMÍA GLOBAL}

Si bien es cierto que la escuela neoclásica continúa siendo el cuerpo teórico dominante en el manejo de la economía actual, también es cierto que esta escuela aún no logra integrar de una manera coherente el financiamiento con la forma como opera la economía capitalista del siglo XXI.

Continuamente se valora el futuro con una visión intertemporal del proceso de Inversión con el fin de aplicar los recursos presentes. De este modo cobra sentido la tasa de interés como instrumento de actualización de los proyectos de inversión, la misma que depende de las condiciones de estabilidad de la economía. Así, a medida que se tenga duda sobre el desempeño futuro de la economía, la tasa de interés será más elevada o viceversa.

De otro lado, para la escuela neoclásica, la hoja de balance de la empresa (en donde los activos son financiados por los pasivos y el capital) no cuenta. La hoja de balance sirve para considerar un esquema de financiamiento proveniente de la capacidad de endeudamiento de la empresa y de sus recursos internos. De tal modo que la masificación del crédito ocurrida en el siglo XX y en lo que va del siglo XXI ha resultado ser sumamente heterogénea a nivel internacional, dependiendo de la norma contable y de la capacidad de endeudamiento de las empresas.

En los países subdesarrollados la capacidad de endeudamiento no responde a una evaluación de los flujos futuros de las empresas (evaluación de proyectos), sino que se basa más que nada en las garantías que pueden ofrecer estas empresas. Esto ha conducido al sistema de financiamiento al absurdo de prestar al que tiene recursos y no al que los necesita para desarrollar sus proyectos. Es así como la banca comercial en los países en desarrollo es más de carácter corporativo y se encuentra enfocada en los grandes negocios. El pequeño negocio ha sido relegado y se atiende en la visión de aumentar la captación, tratando de no incurrir en riesgos y ofreciendo montos de crédito que lo limitan, dándose en el esquema de financiamiento actual la paradoja de que el sistema financiero alienta a una concentración de las carteras de crédito, al realizar transferencias en- 
tre grupos sociales, captando de los grupos de menores ingresos y financiando a las empresas y a los estratos de más altos ingresos ${ }^{20}$.

De otro lado, si se tiene cuenta el cambio del viejo modelo de sustitución de importaciones a uno de apertura, que implica el paso del primero basado en la maximización de utilidades vía precio al otro basado en el volumen, la nueva cultura empresarial debe basar sus operaciones en las economías de escala para poder competir en la economía global, lo cual significa que el nuevo modelo es demandante de recursos financieros para poder lograr economías de escala que le permitan competir en el mundo global. Por otro lado, el ajuste de los precios a niveles internacionales demanda también un aumento de las necesidades de financiamiento que no pueden atenderse por la vía del autofinanciamiento.

Los países en desarrollo cuentan con empresas que en promedio tienen relaciones pasivo-capital por debajo de los estándares internacionales. Para aumentar el capital (parejo a los pasivos) se requiere fortalecer los flujos de efectivo por parte de la empresa, los cuales dependen del crecimiento de la demanda agregada. A fin de garantizar un crecimiento sostenido de dicha demanda y con ello asegurar el aumento de los flujos de efectivo, los países en desarrollo requieren buscar una expansión uniforme de todos los sectores de la economía. Asimismo, para que los flujos de efectivo se reinviertan se requiere establecer una política tributaria que premie la reinversión de utilidades y castigue el consumo de ganancias.

En la economía global la expansión del capital depende también de la expansión de los mercados de valores, que juegan un papel importante en el paso de la empresa familiar a empresa pública por la vía de colocación de acciones. En los países en desarrollo la mayor parte de las empresas sigue conservando su carácter familiar y son pocas las que se financian por la emisión de acciones. Todo lo contrario, el sistema de mercado que se está consolidando, en vez de incorporar al rango de micro y pequeñas empresas en el esquema de financiamiento, las margina, debido a que el esquema de garantías que solicita la banca comercial hace casi imposi- ble el acceso a sus recursos por parte de empresas poco capitalizadas.

De modo que la única alternativa que se visualiza teóricamente para el financiamiento del crecimiento en los países del tercer mundo es la banca de desarrollo, la cual podría ser una opción para otorgar las garantías necesarias de forma que las instituciones del mercado formal pudieran ofrecer financiamiento a las micro y pequeñas empresas poco capitalizadas y que actualmente se encuentran marginadas del mercado. Tal vez ésta sea la tarea más importante en materia de financiamiento del desarrollo, puesto que este grupo de empresas en términos de establecimientos son las que representan la mayor parte del sector empresarial en el mundo en desarrollo.

Tomando en cuenta el nuevo ambiente que genera la globalización financiera y la realidad financiera de los países en desarrollo, el Banco Central de estos países asume un papel prioritario en procura de los objetivos nacionales de crecimiento sostenido y estabilidad del poder adquisitivo de la moneda, para lo cual debe mantener una política monetaria que otorgue liquidez suficiente al sistema.

Los países en desarrollo requieren definir claramente el tipo de sistema financiero que desean tener de acuerdo a su realidad empresarial. Sin embargo, estos países han tenido problemas para definir este esquema, por ello cuentan con sistemas financieros incompletos e insuficientes para financiar el desarrollo de manera adecuada.

En el caso de América Latina no se ha logrado desarrollar un sistema tributario eficiente y todavía mantienen bajas cargas fiscales, lo que ha impedido sostener un ritmo adecuado de crecimiento del gasto público. En este sentido, se dice que a América Latina le falta madurez institucional, la cual es necesaria en el complejo mundo de la globalidad financiera. De tal forma que el Banco Central surge como un esquema complementario a los ministerios de economía y finanzas, los mismos que para financiar sus gastos mantienen como caja a los Bancos Centrales $^{21}$. A su vez el resultado de la falta de madurez institucional es una débil estructura fiscal favorecedora de la concentración del ingreso y con una carga tributaria no equitativa. 


\section{LA INDEPENDENCIA DE LA BANCA CENTRAL EN AMÉRICA LATINA}

Con respecto a la independencia de la banca central en América Latina cabe mencionar que ésta mantiene tal estatus a partir de la reforma legal adoptada en los años noventa. Sin embargo, se ha demostrado que existe una gran brecha entre independencia de jure e independencia de facto de los bancos centrales de América Latina, a propósito de establecer una correlación altamente significativa entre inflación e independencia efectiva, utilizando como indicador de esta última, la tasa de rotación de gobernadores o presidentes de los Bancos Centrales ${ }^{22}$. Asimismo, se ha demostrado también la no existencia de correlación entre inflación e independencia legal ${ }^{23}$.

La mayor independencia legal que se asignó a los Bancos Centrales en los noventa, tiene por finalidad generar una mayor autonomía en el manejo de los instrumentos de la política monetaria, en la búsqueda de la estabilidad de los precios. Asimismo, limitar la monetización del déficit de los gobiernos. Sin embargo, se ha demostrado que son menores los avances logrados en la concesión de autonomía política a los Bancos Centrales de la región, en la medida que en un número importante de países los gobiernos mantienen la facultad de remover directamente al gobernador o presidente del Banco Central, lo cual es coherente con el alto grado de rotación de los gobernadores de Bancos Centrales durante los noventa en varios países de América Latina, al haber sido retirados con anterioridad a los períodos fijados en la carta orgánica de los Bancos Centrales ${ }^{24}$.

\section{CONCLUSIONES}

1. En los países desarrollados la mayoría de la fuerza laboral tiene el nivel de competitividad adecuado a los estándares internacionales, mientras que los niveles de competitividad de los países en desarrollo guardan un rezago en relación a dichos estándares, que se elevan continuamente con los avances de la tecnología.

2. La reinserción de las economías en desarrollo a la economía global puede ser el eje en torno al cual se desplieguen oportunidades de desarrollo nacional, que en la actualidad dependen de la articulación nacional a la producción mundial integrada o de las ca- denas productivas, donde los conceptos de coordinación y aprendizaje ocupan un lugar central en el estudio de la reinserción internacional.

3. La inserción territorial en segmentos específicos de la cadena de valor no es tan sencilla; se considera necesario generar condiciones competitivas para el sector productivo de las economías, a partir de los cuales se generan oportunidades y retos sistémicos de políticas que van mucho más allá de las políticas a nivel macro, micro, meso y meta económicas, en concordancia con un concepto aún más complejo como es el de la competitividad global.

4. La capacidad de los territorios de las economías en desarrollo de endogeneizar los procesos y segmentos de cadenas mediante los cuales se integran al mercado mundial, son de la mayor trascendencia en aspectos relacionados con la investigación y desarrollo y su difusión; la generación de valor agregado y de empleo y su calidad; los procesos de aprendizaje tecnológico; el nivel de capacitación y educación, entre otras variables socioeconómicas.

5. Los efectos del proceso de globalización en términos socioeconómicos y territoriales pueden generar los denominados procesos de endogeneidad territorial o de polarización, que dependen de las condiciones financieras estables que brinden autonomía a la gestión macroeconómica de las economías nacionales.

6. Los movimientos de capital que vienen acompañando a la globalización financiera se asocian con una mayor inestabilidad en las economías en desarrollo, y se ha aceptado que una mayor apertura financiera puede restringir la autonomía de la gestión macroeconómica para aplicar medidas de políticas económicas nacionales apropiadas, dado que los movimientos de capital que han acompañado a la globalización financiera se han asociado con una mayor inestabilidad por lo especulativo de la inversión de cartera, que es capaz de desatar una crisis en cualquier país que no observe la disciplina financiera que impone la globalización.

7. Si bien es cierto que la escuela neoclásica continúa siendo el cuerpo teórico dominante 
en el manejo de la economía actual, también es cierto que esta escuela aún no logra integrar de una manera coherente el financiamiento con la forma como opera la economía capitalista del siglo XXI.

8. En la economía global la expansión del capital depende también de la expansión de los mercados de valores, que juegan un papel importante en el paso de la empresa familiar a empresa pública por la vía de colocación de acciones. En los países en desarrollo la mayor parte de las empresas sigue conservando su carácter familiar y son pocas las que se financian por la emisión de acciones. Todo lo contrario al sistema de mercado que se está consolidando, que en vez de incorporar al rango de micro y pequeñas empresas en el esquema de financiamiento, las margina, debido a que el esquema de garantías que solicita la banca comercial hace casi imposible el acceso a sus recursos por empresas poco capitalizadas.

9. Teóricamente, una alternativa viable para el financiamiento del crecimiento en los países del tercer mundo es la banca de desarrollo, la cual podría ser una opción para otorgar las garantías necesarias de forma que las instituciones del mercado formal pudieran ofrecer financiamiento a las micro y pequeñas empresas poco capitalizadas y que actualmente se hallan marginadas del mercado. Tal vez ésta sea la tarea más importante en materia de financiamiento del desarrollo, puesto que este grupo de empresas en términos de establecimientos son las que representan la mayor parte del sector empresarial en el mundo en desarrollo y las que generan empleo.

10. Tomando en cuenta el nuevo ambiente que genera la globalización financiera y la realidad financiera de los países en desarrollo, el Banco Central en estos países asume un papel prioritario en procura de los objetivos nacionales de crecimiento sostenido y la estabilidad del poder adquisitivo de la moneda, para lo cual debe mantener una política monetaria que otorgue liquidez suficiente al sistema

11. De allí que se asigna a los Bancos Centrales una mayor independencia legal a partir de los noventa, a fin de generar una mayor autonomía en el manejo de los instrumentos de la política monetaria, en la búsque- da de la estabilidad de los precios, y de limitar la monetización del déficit de los gobiernos. Sin embargo, se ha demostrado que son menores los avances logrados en la concesión de autonomía política a los Bancos Centrales de la región, en la medida de que en un número importante de países, los gobiernos mantienen la facultad de remover directamente al gobernador o presidente del Banco Central.

\section{NOTAS}

1. Este trabajo fue realizado en el marco del Proyecto de investigación "Globalización e Integración Económica: el TLC con Estados Unidos, retos y perspectivas", desarrollado en el Instituto de Investigación de la Facultad de Ciencias Administrativas (UNMSM), y presentado a la IX Asamblea General de ALAFEC, siendo aprobado por el Comité Ciego Internacional para ser presentado como ponencia en dicha Asamblea, en el área de Negocios Internacionales. La Habana, Cuba.

2. Amin agrupa a los países en: Primer, Tercer y Cuarto Mundo. Amin, Samir. El Capitalismo en la Era de la Globalización. Primera Edición. Barcelona, Editorial Paidós Iberica S.A., p. 2.

3. Manchón, Federico. (1997). La regionalización como dimensionamiento histórico de la gestión macroeconómica. Ponencia presentada en el XXI Congreso de la Asociación Latinoamericana de Sociología. Sao Paulo, septiembre de 1997, p. 1.

4. Álvarez Béjar, Alejandro. (2000). Integración económica, crisis financiera y patrones de especialización industrial en la región Centro-Norte de México: 1994-2000. Ponencia a presentar en el Sexto Congreso Internacional sobre Estudios Regionales (AMECIDER). Cuernavaca - Morelos, CRIM-UNAM, 14 al 16 de junio de 2000.

5. Manchón, Federico. (1999). Globalización económica y finanzas internacionales. En: Estay, Jaime et al (c). (1999). La globalización de la economía mundial. Principales dimensiones en el umbral del siglo XXI. México, Universidad Nacional Autónoma de México, Instituto de Investigaciones Económicas, Benemérita Universidad Autónoma de Puebla, Centro de Investigaciones de la Economía Mundial y Miguel Ángel Porrúa.

6. Villarreal, René.

7. Dussel Peters, Enrique y Liu Xue, Dong. (2004). Oportunidades y retos económicos de China para México y Centroamérica. México, CEPAL, Naciones Unidas.

8. Gereffi y Korzeniewicz (1994); Piore y Sabel (1984); UNCTAD (2001), (2003). En: Dussel Peters y Liu Xue. Op. Cit., p. 2.

9. Storper (1997). En: Dussel, Peters y Liu Xue, Dong. Op. cit., p. 3.

10. Bair y Dussel, Peters (2004), citado en: Dussel, Peters y Liu Xue, Dong. Op. cit., p. 4.

11. La eficiencia colectiva es comprendida como la ventaja competitiva resultante de externalidades de economías locales y acciones conjuntas. Este concepto viene siendo analizado desde la segunda mitad de los noventa por autores como Humphrey y Schmitz (2001), citados en Dussel, Peters y Liu Xue, Dong (2004). Op. cit., p. 4. 
12. Ernst (2003:2). En: Dussel, Peters y Liu Xue, Dong. (2004). Op. cit., p. 4.

13. Chang (2002); ATS y Stumpo (2001); Lall (1999). En: Dussel, Peters y Liu Xue, Dong. Op. cit., p. 5.

14. Rodrick (2003:10-11). En Dussel, Peters y Liu Xue, Dong (2004). Op. cit., p. 5.

15. Dussel P. (2004); Messner (2002); Messner y MeyerStamer (1994). En Dussel, Peters y Liu Xue, Dong (2004). Op. cit., p. 6.

16. Gereffi; Humphrey; Kaplinsy y Sturgeon (2001). En Dussel, Peters y Liu Xue, Dong. (2004). Op. cit., p. 6.

17. Dussel, Peters y Liu Xue, Dong (2004). Op. cit., p. 6.

18. Al respecto, Naciones Unidas ha puesto en discusión la introducción de un impuesto a los movimientos de capital de corto plazo con el fin de reducir la inestabilidad financiera, tal como lo plantea el Premio Nobel James Tobin. Ruiz D., Clemente. (1999). Macroeconomia Global, Fundamentos Institucionales y de Organización Industrial. Primera Edición. México, UNAM y Edit. Jus, S.A. de CV, p. 7.

19. Huerta, Arturo. Globalización causa /crisis asiática. Instituto de Investigaciones Económicas, Facultad de Economía, UNAM.

20. Ruiz D., Clemente. (1999). Macroeconomia Global, Fundamentos Institucionales y de Organización Industrial. Primera Edición. México, UNAM y Edit. Jus, S.A. de CV.

21. El modelo de Programación Financiera supone que un exceso de gasto público trae como consecuencia el aumento del crédito al gobierno de parte del Banco Central. Esto conduce a un aumento del crédito interno por encima del saldo de la base monetaria, dando como resultado la disminución de las reservas internacionales netas, precipitándose de este modo la crisis de balanza de pagos. En Ruiz D., Clemente. Op.cit., p. 189.

22. Jácome utiliza el índice de Cukierman modificado en base a criterios que han adquirido relevancia en los años recientes en la discusión respecto a buenas prácticas en el establecimiento de la autonomía de los Bancos Centrales. Jácome H., Luis I. (2003). Independencia legal de la banca central en América Latina e inflación. México, El Trimestre Económico, Volumen LXX (4), № 280, FCE, octubre-Diciembre de 2003, p. 690.

23. Jácome utiliza el índice de Cukierman para cuantificar el grado de independencia legal de 17 países de América Latina. Jácome (2003). Op. Cit., p. 689.

24. Ibid., pp. 701-702.

\section{BIBLIOGRAFÍA}

Álvarez Béjar, Alejandro. (2000). Integración económica, crisis financiera y patrones de especialización industrial en la región Centro-Norte de México: 1994-2000. Ponencia para presentar en el Sexto Congreso Internacional sobre Estudios Regionales (AMECIDER). Cuernavaca - Morelos, CRIM-UNAM, 14 al 16 de junio del 2000.

Amin, Samir. El Capitalismo en la era de la Globalización. Primera Edición. Barcelona, Editorial Paidós Iberica S.A.
Dussel Peters, Enrique y Liu Xue, Dong. (2004). Oportunidades y retos económicos de China para México y Centroamérica. México, CEPAL, Naciones Unidas.

Huerta, Arturo. Globalización causa /crisis asiática. Instituto de Investigaciones Económicas, Facultad de Economía, UNAM.

Humphrey, John. (2000). Assembler-Supplier Relations in the Auto Industry: Globalization and National Development. En Competition $\&$ Change. Singapore, OPA.

Jácome H., Luis I. (2003). Independencia legal de la banca central en América Latina e inflación. En El Trimestre Económico, Volumen LXX(4), № 280. México, FCE, octubre-diciembre del 2003.

Manchón Federico. (1997). La regionalización como dimensionamiento histórico de la gestión macroeconómica. Ponencia presentada en el XXI Congreso de la Asociación Latinoamericana de Sociología, Sao Paulo, setiembre de 1997. Ver: http://www.redem. buap.mx/federico.htm.

Manchón Federico. (1999). Globalización económica y finanzas internacionales. En Estay, Jaime et al (c). (1999). La globalización de la economía mundial. Principales dimensiones en el umbral del siglo XXI. México, Universidad Nacional Autónoma de México, Instituto de Investigaciones Económicas, Benemérita Universidad Autónoma de Puebla, Centro de Investigaciones de la Economía Mundial y Miguel Ángel Porrúa. En http://www.redem.buap.mx/acrobat/ manchon2.pdf

Naciones Unidas. (2003). UNCTAD/TDR/2003. Conferencia de las Naciones Unidas sobre comercio y desarrollo, la acumulación de capital, el crecimiento económico y el cambio estructural. Nueva York et Ginebra, Naciones Unidas.

Naciones Unidas. (2004). UNCTAD/TDR/2004. Trade and Develpment Report, Policy coherence Development strategies and integration into the world economy. Nueva York et Ginebra, United Nations.

Rivera, Miguel Angel. (2001). México en la economía global: reinserción, aprendizaje y coordinación. En Revista Problemas del Desarrollo, $N^{\circ} 127$, octubre/diciembre de 2001.

Ruiz D., Clemente. (1999). Macroeconomia Global, Fundamentos Institucionales y de Organización Industrial. Primera Edición. México, UNAM y Edit. Jus S.A. de CV. 